\title{
A case report of heterotopic pregnancy
}

\section{Keerthana R.*, Sundar Narayanan S.}

Department of Endogynecology, Sudha Sundar Hospital, Putheri, Kanyakumari, Tamil Nadu, India

Received: 13 November 2021

Accepted: 02 December 2021

\author{
*Correspondence: \\ Dr. Keerthana R., \\ E-mail: drkeerthanaradhakrishnan@gmail.com
}

Copyright: (c) the author(s), publisher and licensee Medip Academy. This is an open-access article distributed under the terms of the Creative Commons Attribution Non-Commercial License, which permits unrestricted non-commercial use, distribution, and reproduction in any medium, provided the original work is properly cited.

\begin{abstract}
Heterotopic pregnancy is the presence of both intrauterine and extrauterine (ectopic) implantation as described by Reece in 1983 and is extremely rare. It accounts for 1 per 30000, in natural cycles and 9 per 10000, in assisted reproduction cycles. The aim of this report is to introduce this case as it poses a challenge to diagnosis due to its complex clinical and laboratory findings. A primigravida aged 30 at 6 weeks period of gestation, reported with minimal bleeding per vaginum. On performing a physical examination her vitals were stable and no significant findings were noted except for an enlarged uterus corresponding to 6 weeks with posterior forniceal fullness. Her beta-hCG was $23765 \mathrm{IU} / \mathrm{ml}$ and ultrasound showed a live intrauterine gestation with left adnexal mass- likely ectopic gestational sac. Laparoscopy showed a left tubal pregnancy and salpingectomy was done. The postoperative period was uneventful and she was discharged with a single live intrauterine gestation of 6-7 weeks. She carried on with her pregnancy and delivered a healthy baby at term. The diagnosis is possible only in cases when there is a high index of suspicion by the treating clinician. The adnexa must be inspected carefully in the confirmatory ultrasound. The early timely diagnosis gives a good maternal outcome and hence crucial in the management.
\end{abstract}

Keywords: Ectopic, Heterotopic, Infertility, Laparoscopy, Salpingectomy, Pregnancy

\section{INTRODUCTION}

Heterotopic pregnancy was first described by Reece in 1983. ${ }^{1}$ He defined it as the presence of both intrauterine and extrauterine (ectopic) implantation. It is extremely rare as it accounts for 1 per 30000 , in natural cycles. With the advent of advanced assisted reproductive techniques over the past few decades, its incidence shows an increasing trend and stands at 9 per 10000 cases. $^{2}$ The aim of the case report was to show that timely diagnosis helps in better management and gives a favourable outcome.

\section{CASE REPORT}

A primigravida aged 30 at 6 weeks period of gestation after spontaneous conception came with complaints of bleeding per vaginum for one day duration associated with abdominal pain. She has regular menstrual cycles with no associated comorbidities or surgeries. On admission her vital signs were satisfactory. Her pulse rate was 84 beats per min, blood pressure 130/70 $\mathrm{mmHg}$ with normal temperature. Her physical examination showed normal systemic examination with mild tenderness over the hypogastric region. In gynecological examination there was brownish discharge on per speculum examination and bimanual findings were normal except for an enlarged uterus of 6 weeks size with posterior forniceal fullness. Her laboratory tests were within normal limits. Her betahCG level was $23765 \mathrm{mIU} / \mathrm{ml}$. Transvaginal ultrasound was done and it showed the following features: (a) single live intrauterine fetus corresponding to 6 weeks noted. Decidual reaction is good and fetal cardiac activity seen; (b) left adnexa- an illdefined heteroechoeic mass with a thick walled complex cystic component $28 \times 16 \mathrm{~mm}$ with peripheral vascularity noted- likely ectopic gestational sac; (c) free fluid in pouch of Douglas; and (d) right ovarycorpus luteal cyst noted. Diagnosis of heterotopic pregnancy was made and laparoscopy was decided with consent of the patient and bystanders. Informed consent was obtained and laparoscopy proceeded. Intraoperatively uterus appeared bulky. Right tube and both ovaries were 
normal. Left tube showed an unruptured tubal ectopic gestation with features of tubal abortion noted and the tube appeared sacculated and edematous. The pouch of Douglas had minimal hemoperitoneum which was suctioned out. Left laparoscopic salpingectomy was done and specimen was sent for histopathology. The histopathological report confirmed the diagnosis. The post-operative period was uneventful and she got discharged on second postoperative day with ultrasound showing a viable intrauterine pregnancy of 6-7 weeks. Her antenatal period was normal and she delivered a live term healthy baby.

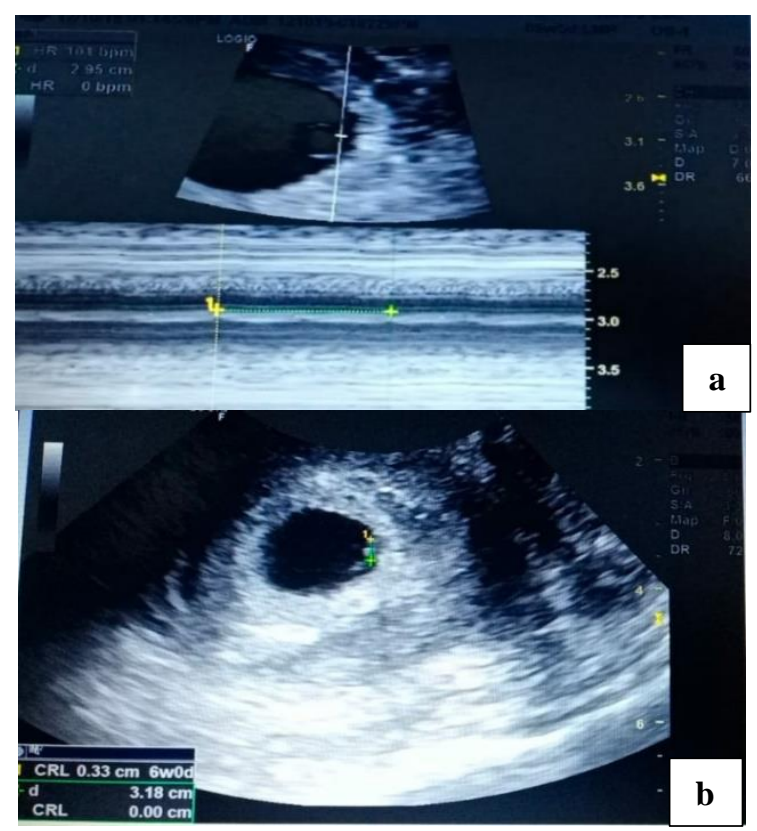

Figure 1: (a) Shows single viable intrauterine gestational sac with cardiac activity; and (b) the crown rump length corresponding to 6 weeks period of gestation of the reported patient.

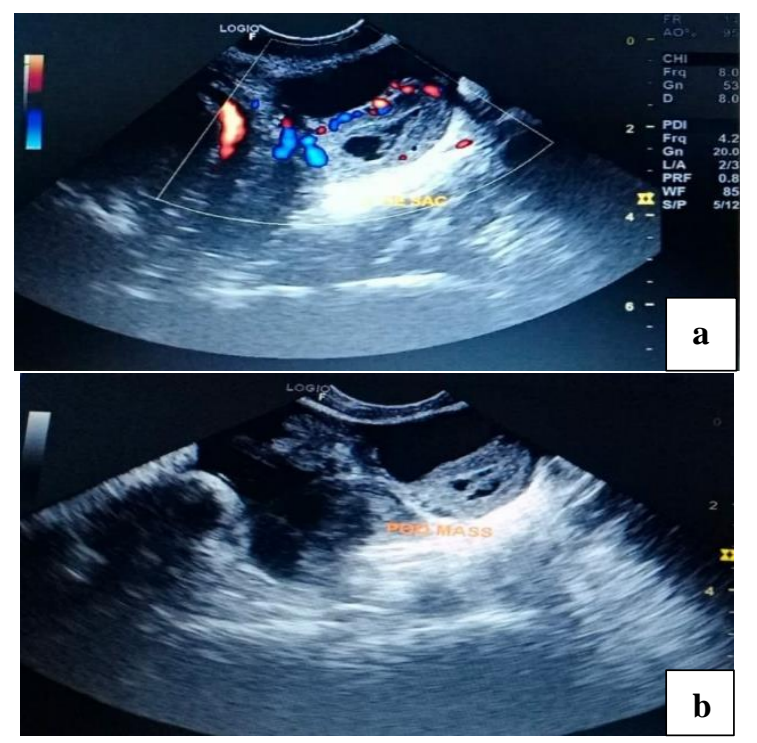

Figure 2: Shows left adnexal complex cystic mass- illdefined with ring of vascularity; and (b) noted in pouch of Douglas of the reported patient.

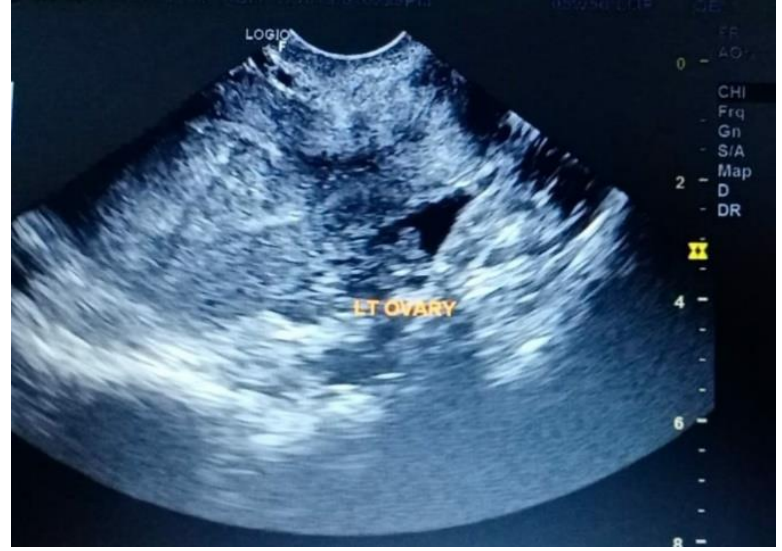

Figure 3: Shows a normal left ovary separate from the adnexal mass of the reported patient.

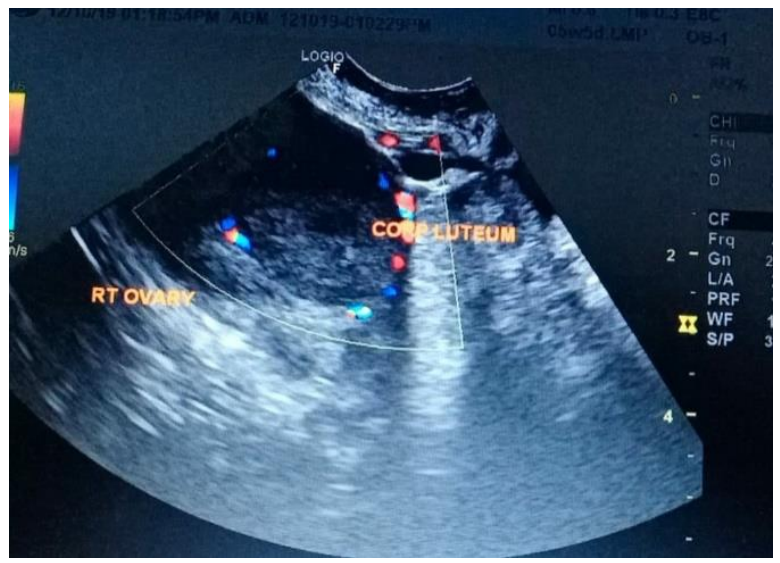

Figure 4: Shows right ovary with corpus luteal cyst of the reported patient.

\section{DISCUSSION}

Reece et al reported the condition first and it included cases conceived spontaneously. ${ }^{1}$ The condition is extremely rare. But in the millennium years with considerable development of infertility treatment, the incidence is showing a rising trend. ${ }^{2}$ The major risk factors for heterotopic pregnancy are chronic pelvic inflammatory disease, use of intrauterine disease, previous history of ectopic, previous tubal surgeries, assisted reproductive techniques. $^{3,4}$ The causes attributed to an independent ectopic gestation most likely is also considered as a risk factor for heterotopic pregnancy. The most frequent location are the fallopian tubes followed by the ovaries. There are many more case reports to show the locations in cervix, peritoneal cavity and uterine cornua. There is also reported cases of tubal ectopic associated with pathological intrauterine pregnancy like blighted ovum or early intrauterine demise. ${ }^{5}$

Many case reports and studies have thrown light on this topic. But more systematic reviews are required to follow definite consensus for management. The transvaginal ultrasound for confirmation of pregnancy around 4-6 
weeks period of gestation is the best tool for diagnosis. The adnexa should be imaged in the early ultrasound. The quantitative serum beta hCG values are the prime tool for managing ectopic pregnancy as diagnostic and post treatment response indicator. But in case of heterotopic pregnancy the beta hCG values cannot be trusted for diagnosis as there is presence of a viable intrauterine pregnancy. The monitoring of beta hCG post conservative measures also proves difficult in this case.

The many conservative measures described by various authors are local methotrexate injection (a minimal of systemic absorption is noted as risk to the developing intrauterine fetus), local $\mathrm{KCl}$ injection (resulted in hematosalpinx), placing hematostatic sutures, prostaglandins (causes teratogencity). ${ }^{6}$ Many other studies support conservative management with favorable outcomes. Laparoscopic salpingectomy is considered as a standard approach in a study done in China with 25 patients with heterotopic pregnancy. ${ }^{7}$ More evidence is required for conclusive guidelines. Around 70-80 percent of patients with prompt diagnosis and treatment carried on the pregnancy till term. If untreated it leads to a mortality of 1 percent. ${ }^{3}$ Hence early timely diagnosis and prompt intervention can make a lot of difference in the outcome. Heterotopic pregnancy is possible with natural conception and the continuation of the pregnancy is feasible with a favourable outcome. ${ }^{8-10}$

\section{CONCLUSION}

The diagnosis is possible only in cases when there is a high index of suspicion by the treating clinician. The clinical presentation is like that of an ectopic pregnancy where the patient presents with abdominal pain and bleeding per vaginum. The adnexa must be inspected carefully in the confirmatory ultrasound. The early timely diagnosis gives a good maternal outcome and hence crucial in the management.

Funding: No funding sources Conflict of interest: None declared Ethical approval: Not required

\section{REFERENCES}

1. Elnahry AG, Elnahry GA. Management of Idiopathic Intracranial Hypertension During the COVID-19 Pandemic. Rev Recent Clin Trials. 2021;16(2):122-5.

2. Walker DJ, Clarke TC, Kennedy CR. Heterotopic ectopic and intrauterine pregnancy after embryo replacement. $\mathrm{Br} \quad \mathrm{J}$ Obstet Gynaecol. 1993;100(11):1048-9.

3. Tal J, Haddad S, Gordon N, Tritsch I. Heterotopic pregnancy after ovulation induction and assisted reproductive technologies: a literature review from 1971 to 1993. Fertil Steril. 1996;66(1):1-12.

4. Wygledowski J, Sawicki W, Cendrowski K. Case of co existent intra and extra uterine pregnancy. Med sci Monit. 1998;4:522-5.

5. Dundar O, Tutuncu L, Mungen E, Muhcu M, Yergok YZ. Heterotopic pregnancy: tubal ectopic pregnancy and monochorionic monoamniotic twin pregnancy: a case report. Perinatal J. 2006;14:96-100.

6. Wright A, Kowalczyk CL, Quintero R, Leach RE. Selective embryo reduction in a heterotopic pregnancy using potassium chloride injection resulting in a hematosalpinx. Fertil Steril. 1996;66(6):1028-30.

7. Yu Y, Xu W, Xie Z, Huang Q, Li S. Management and outcome of 25 heterotopic pregnancies in Zhejiang, China. Eur J Obstet Gynecol Reprod Biol. 2014;180:157-61.

8. Tingi E, Rowland J, Hanna L. A case of heterotopic pregnancy following spontaneous conception. J Obstet Gynaecol. 2015;35(4):430-1.

9. Varras M, Akrivis C, Hadjopoulos G, Antoniou N. Heterotopic pregnancy in a natural conception cycle presenting with tubal rupture: a case report and review of the literature. Eur J Obstet Gynecol Reprod Biol. 2003;106(1):79-82.

10. Jan F, Naikoo GM, Rather MH, Sheikh TA, Rather YH. Ruptured heterotopic pregnancy: a rare cause for hemoperitoneum; report of three cases from Kashmir, India. Indian J Surg. 2010;72(5):404-6.

Cite this article as: Keerthana R, Sundar NS. A case report of heterotopic pregnancy. Int J Reprod Contracept Obstet Gynecol 2022;11:269-71. 